\title{
Evaluation of intraoperative and postoperative complications related to lymphadenectomy in ovarian cancer patients
}

\author{
LESZEK JAROSLAW GMYREK ${ }^{1}$, JOANNA JONSKA-GMYREK ${ }^{2}$, \\ PIOTR SOBICZEWSKI ${ }^{1}$, GRZEGORZ PANEK ${ }^{1}$ and MARIUSZ BIDZINSKI ${ }^{1}$ \\ Departments of ${ }^{1}$ Gynecological Oncology and ${ }^{2}$ Radiotherapy, \\ Maria Sklodowska-Curie Memorial Cancer Centre, Warsaw, Poland
}

Received November 12, 2010; Accepted March 1, 2011

DOI: $10.3892 / \mathrm{ol} .2011 .281$

\begin{abstract}
Assessment of the regional lymph node status is an integral part of diagnostics for ovarian cancer patients. Due to the risk of complications, lymphadenectomy for ovarian cancer patients, as a routine treatment procedure, is still a subject of controversy. Therefore, the present study aimed to evaluate the frequency and nature of intraoperative and postoperative complications in ovarian cancer patients treated with surgery. In addition, a comparison of the frequency and nature of surgical complications between patients who underwent lymphadenectomy and those who did not was carried out. A retrospective analysis of 211 consecutive ovarian cancer patients treated with surgery with pelvic and aortic lymphadenectomy (group I), and 258 ovarian cancer patients treated with surgery but without lymphadenectomy (group II) was carried out. All of the patients were treated with complementary chemotherapy. The frequency and nature of the intraoperative and postoperative complications were determined. The most frequent intraoperative complications in the two groups were haemorrhage, urinary system damage and digestive tract damage. The difference in the frequency and nature between the groups was not statistically significant $(\mathrm{p}=0.683)$. The most frequent postoperative complications were haemorrhage, intestinal junction dehiscence, eventrations, wound dehiscence, anaemia, wound healing complications and intestinal fistulas. Haemorrhage, eventrations and wound healing complications were more frequent in group I. The difference was statistically significant $(\mathrm{p}=0.002)$. Due to postoperative complications, reoperative procedures were necessary in two women in group II (0.78) and in 15 women $(7.11 \%)$ in group I. The difference was statistically significant $(\mathrm{p}=0.000)$. In conclusion, the most frequent intraoperative complications were haemorrhage, urinary system damage and digestive tract damage. The frequency of
\end{abstract}

Correspondence to: Dr Joanna Jonska-Gmyrek, Department of Gynecological Oncology, Maria Sklodowska-Curie Memorial Cancer Centre, Walczyka Street 39, 02-849 Warsaw, Poland E-mail: jonska@wp.pl

Key words: ovarian cancer, lymphadenectomy, treatment complications complication was found to be similar in the two groups. A statistically significant higher rate of postoperative complications, such as haemorrhage, eventrations and wound healing complications was confirmed in the lymphadenectomy group.

\section{Introduction}

Worldwide epidemiological studies indicate a rising trend in ovarian cancer morbidity. The number of new ovarian cancer cases worldwide was estimated at 192,000 in 2001, which accounts for $4.0 \%$ of all types of cancer among women. The mortality rate of ovarian cancer is estimated at 114,000 individuals per year (1).

Assessment of regional lymph node involvement is an integral part of the diagnostic procedure for ovarian cancer patients. Due to the risk of complications related to this procedure, the routine performance of lymphadenectomy in ovarian cancer patients is still the subject of controversy. Therefore, the present study aimed to evaluate the frequency and nature of intraoperative and postoperative complications in ovarian cancer patients treated with surgery, as well as compare the frequency and nature of surgical complications between a group of patients who underwent lymphadenectomy and a group who did not undergo this procedure.

\section{Materials and methods}

A retrospective analysis of 211 consecutive ovarian cancer patients (group I) treated at the Gynecological Oncology Department, Maria Sklodowska-Curie Memorial Cancer Centre, between 1998 and 2006 was carried out. The patient characteristics are shown in Table I. The patients underwent surgery according to routine surgical-pathological protocol with pelvic and aortic lymphadenectomy. During the same study period, a group of 258 ovarian cancer patients was also treated with surgery but without lymphadenectomy (group II). The majority of patients in group II were treated surgically at other centers and came to our department to receive adjuvant treatment. Complementary chemotherapy based on taxanes and cisplatin was administered to all of the patients.

Assessment of the correlations between the two factors was carried out using the Pearson's correlation analysis for factors, with a normal distribution. In case of differences 
Table I. Patient characteristics.

\begin{tabular}{|c|c|c|c|}
\hline Characteristics & $\begin{array}{l}\text { Group I } \\
(\mathrm{n}=211)\end{array}$ & $\begin{array}{l}\text { Group II } \\
(\mathrm{n}=258)\end{array}$ & p-value \\
\hline \multicolumn{4}{|l|}{ Age, in years } \\
\hline Mean (range) & $55(20-81)$ & $53(21-79)$ & 0.48 \\
\hline \multicolumn{4}{|l|}{ FIGO stage, n (\%) } \\
\hline I-II & $76(36.0)$ & $91(35.0)$ & 0.40 \\
\hline III-IV & $135(64.0)$ & $167(65.0)$ & \\
\hline \multicolumn{4}{|l|}{ Histopathology, n (\%) } \\
\hline Serous & $107(50.7)$ & $138(53.5)$ & 0.66 \\
\hline Mucinous & $22(10.4)$ & $21 \quad(8.0)$ & \\
\hline Endometrioid & $44(20.8)$ & 48 (18.5) & \\
\hline Clear cell & $15 \quad(7.2)$ & $20(7.6)$ & \\
\hline Other (mixed, non-differentiated) & $22(10.4)$ & $31(12.0)$ & \\
\hline Missing data & $1 \quad(0.5)$ & $1(0.4)$ & \\
\hline \multicolumn{4}{|l|}{ Grade, n (\%) } \\
\hline G1 & $22(10.4)$ & $22(8.4)$ & 0.90 \\
\hline $\mathrm{G} 2$ & $78(37.0)$ & $96(38.1)$ & \\
\hline G3 & $88(41.7)$ & $110(42.6)$ & \\
\hline GX & $19(9.0)$ & $28(10.8)$ & \\
\hline Missing data & $4 \quad(1.9)$ & $2(0.8)$ & \\
\hline \multicolumn{4}{|l|}{ WHO status, n (\%) } \\
\hline 0 & $156(73.9)$ & $172(66.7)$ & 0.25 \\
\hline $1-2$ & $55(26.1)$ & $83(33.3)$ & \\
\hline
\end{tabular}

other than a normal distribution, variables were expressed as median, interval, and upper and lower quartile.

A multivariate analysis was performed to assess the effect of the analyzed factors on overall survival (OS) and diseasefree survival (DFS) using Cox's regression model. $\mathrm{P}=0.05$ was considered to be of clinical significance. The factors evaluted for multivariate analysis were: age, the pretreatment performance status according to the WHO classification, clinical stage of the disease evaluated according to the FIGO classification, the type of surgical treatment (lymphadenectomy vs. no lymphadenectomy), pretreatment size of the tumor, size of retroperitoneal lymph nodes, ascites, the presence of cancer cells in peritoneal washings, serum level of CA125 tumor marker after the third course of chemotherapy in reference to the norm, size of residual disease, number of excised and positive pelvic and aortic lymph nodes, histology and tumor grade.

Using the $\chi^{2}$ test, an analysis of the frequency and type of intraoperative and postoperative complications was carried out. A comparison of the type and frequency of surgery-related complications between the group with lymphadenectomy and without this procedure was then carried out.

\section{Results}

The median follow-up was 40.56 (7.08-139.2) months. The FIGO stages in group I were I-II in 76 (36) patients and III-IV in $135(64 \%)$ patients. The 5-year OS in this group was 94 and $31 \%$, respectively. The FIGO stages in group II were FIGO I-II in 91 (35.0) patients and FIGO III-IV in 167 (65.0\%) patients. The 5-year OS in this group was 76 and $25 \%$, respectively. Following a comparison of the survival curves between the two groups, it was shown that the difference in OS in early ovarian cancer (FIGO I, II) was statistically significant $(\mathrm{p}=0.01$ ), while in advanced stages (FIGO III, IV) the difference did not reach statistical significance $(\mathrm{p}>0.1)$. The number and percentage of optimal operations with a residual tumor diameter of $\leq 1 \mathrm{~cm}$ in groups I and II were $189(89.6 \%)$ and $240(93 \%)$, respectively.

The most frequent intraoperative complications in the two groups were haemorrhage, urinary tract damage and digestive tract damage. The difference between the number of complications between the groups was not statistically significant $\left(\chi^{2}=2.2876\right)(\mathrm{p}=0.683)$ (Table II).

The most frequent postoperative complications in the two groups were abdominal haemorrhage, intestinal junction dehiscence, intestinal fistula, urinary tract fistula, eventration, anaemia, wound healing complication, postoperative fever $>38^{\circ} \mathrm{C}$ and wound infection.

Abdominal haemorrhage, eventration and wound healing complications were the most common postoperative complications in the lymphadenectomy group. The difference in the frequency of the complications between the groups was statistically significant $\left(\chi^{2}=26.1797\right)(\mathrm{p}=0.002)$ (Table III).

Due to postoperative complications, reoperative procedures were necessary in $2(0.78)$ patients of group II and in $15(7.11 \%)$ patients of group I. The difference in the frequency 
Table II. Intraoperative complications $(\mathrm{p}=0.683)$.

\begin{tabular}{lrrr}
\hline Intraoperative complications & $\begin{array}{c}\text { Group I } \\
\mathrm{n}(\%)\end{array}$ & $\begin{array}{c}\text { Group II } \\
\mathrm{n}(\%)\end{array}$ & $\begin{array}{c}\text { Total cases } \\
\mathrm{n}(\%)\end{array}$ \\
\hline No complications & $201(95.26)$ & $246(95.35)$ & $447(95.31)$ \\
Haemorrhage & $4(1.90)$ & $4(1.55)$ & $8(1.71)$ \\
Urinary system damage & $5(2.37)$ & $4(1.55)$ & $(1.92)$ \\
Digestive tract damage & $1(0.47)$ & $2(0.78)$ & $3(0.64)$ \\
Other & $0(0.00)$ & $2(0.78)$ & $(0.43)$ \\
Total & $211(100)$ & $258(100)$ & $(100)$ \\
\hline
\end{tabular}

Table III. Postoperative complications $(\mathrm{p}=0.002)$.

\begin{tabular}{|c|c|c|c|}
\hline Postoperative complications & $\begin{array}{c}\text { Group I } \\
\mathrm{n}(\%)\end{array}$ & $\begin{array}{c}\text { Group II } \\
\mathrm{n}(\%)\end{array}$ & $\begin{array}{l}\text { Cases } \\
\mathrm{n}(\%)\end{array}$ \\
\hline No complications & $178(85.17)$ & $250(96.90)$ & $428(91.65)$ \\
\hline Abdominal haemorrhage & $7 \quad(3.35)$ & $1 \quad(0.39)$ & $8(1.71)$ \\
\hline Intestinal junction dehiscence & $2(0.96)$ & $0 \quad(0.00)$ & $2(0.43)$ \\
\hline Intestinal fistulas & $1 \quad(0.48)$ & $0 \quad(0.00)$ & $1 \quad(0.21)$ \\
\hline Urinary tract fistulas & $2(0.96)$ & $0 \quad(0.00)$ & $2(0.43)$ \\
\hline Eventrations & $7 \quad(3.35)$ & $1 \quad(0.39)$ & $8 \quad(1.71)$ \\
\hline Anaemia & $4 \quad(1.91)$ & $3(1.16)$ & $7 \quad(1.50)$ \\
\hline Wound healing complications & $7 \quad(3.35)$ & $2(0.78)$ & $9 \quad(1.93)$ \\
\hline Postoperative fever $>38^{\circ} \mathrm{C}$ & $0 \quad(0.00)$ & $1 \quad(0.39)$ & $1 \quad(0.21)$ \\
\hline Wound infections & $1 \quad(0.48)$ & $0 \quad(0.00)$ & $1 \quad(0.21)$ \\
\hline Missing data & $2(0.90)$ & $0 \quad(0.00)$ & $2(0.40)$ \\
\hline Total & 211 & $258 \quad(100)$ & $(100)$ \\
\hline
\end{tabular}

Table IV. Percentage of reoperative procedures required in group I and II ( $\mathrm{p}=0.000)$.

\begin{tabular}{|c|c|c|c|}
\hline Reoperations & $\begin{array}{c}\text { Group I } \\
\mathrm{n}(\%)\end{array}$ & $\begin{array}{c}\text { Group II } \\
\mathrm{n}(\%)\end{array}$ & $\begin{array}{l}\text { Cases } \\
\mathrm{n}(\%)\end{array}$ \\
\hline No & $196(92.89)$ & $256(99.22)$ & $452(96.38)$ \\
\hline Yes & $15(7.11)$ & $2(0.78)$ & $17 \quad(3.62)$ \\
\hline Total & $211 \quad(100)$ & $258 \quad(100)$ & $469 \quad(100)$ \\
\hline
\end{tabular}

of complications between the groups was statistically significant $\left(\chi^{2}=13.3296\right)(\mathrm{p}=0.000)($ Table IV).

In conclusion, the frequency and nature of the intraoperative complications between the groups with lymphadenectomy, and the group without this procedure were found to be similar. The frequency and nature of the postoperative complications between the lymphadenectomy group and the group without this procedure were statistically significantly different.

\section{Discussion}

Diagnosis and treatment of ovarian cancer patients is a difficult challenge for gynecological oncologists. Ovarian cancer is one of the gynecological types of cancer associated with a poor patient prognosis and is a leading cause of cancerrelated mortality in females $(2,3)$. This unsatisfactory survival of ovarian cancer patients is due to the absence of effective detection methods for early clinical stages of this disease. Ovarian cancer is characterized as an insidious disease often with no clinical or non-specific symptoms. Consequently, approximately $70-85 \%$ of ovarian cancer patients are diagnosed with an advanced clinical stage of the disease, and a permanent cure is achieved in only approximately $15-30 \%$ of patients $(3,4)$.

Unsatisfactory treatment outcome is also the result of the limited effectiveness of routine treatment methods. Numerous investigators address the issue of how to enhance survival in the malignancy which is diagnosed at such advanced stages. 
One treatment option which is the focus of intensive clinical trials is pelvic and aortic lymphadenectomy as part of a routine radical treatment modality in ovarian cancer patients. The diagnostic role of lymphadenectomy, particularly in early stage ovarian cancer patients, has been confirmed (5). Assessment of the regional lymph node status is an integral part of diagnostics for ovarian cancer patients. This is the mainstay of accurate clinical disease stage assessment and appropriate treatment decision-making (5).

According to data presented by Verleye et al (6), pelvic and aortic lymphadenectomy in early ovarian cancer (FIGO I) is a strong prognostic factor that has an impact on overall and disease-free survival.

Lymphadenectomy in ovarian cancer patients, as part of the routine surgical treatment, is indicated in the event of a high number of lymph node metastases present in these patients. In patients where the FIGO stage was ascertained as I or II, during primary assessment according to abdominal organ status, positive lymph nodes were found in 10.6-48.6\% of cases (7), while in advanced stages (III and IV) positive lymph nodes were found in $38-76 \%$ of cases (3). Based on the above data, the percentage of patients for whom early ovarian cancer (FIGO I) instead of advanced cancer (FIGO III) is diagnosed may be as high as $20 \%$. This may result in inadequate treatment decisions and unfavorable treatment outcome.

The therapeutic role of lymphadenectomy in ovarian cancer patients is the subject of various clinical studies. Assessment of its significance is difficult as the presence of other factors may affect the prognosis in this group of patients. In addition, some investigators emphasize the fact that, due to the extensive nature of the procedure, the risk of complications is high, and the decision to execute lymphadenectomy should be taken after weighing both the risk of complications and possible advantages.

Intraoperative complications. The results of the present study showed that the most frequent intraoperative complications in the two groups were haemorrhage, urinary system damage and digestive tract system damage. The difference between the frequency and nature of the complications between the groups was not statistically significant. Due to incomplete data regarding the duration of the surgical procedure and the volume of blood loss in patients who underwent surgery outside our center, the analysis of these factors was not possible.

Maggioni et al (8) reported that a higher risk of intraoperative complications due to lymphadenectomy in ovarian cancer patients is associated with a longer duration of surgery and greater blood loss.

Postoperative complications. The results of the present study showed a statistically significant difference in the frequency and nature of postoperative complications between groups I and II.

The most frequent postoperative complications were abdominal haemorrhage, intestinal junction dehiscence, intestinal fistulas, urinary tract fistulas, eventrations, anaemia, wound healing complications, postoperative fever $>38^{\circ} \mathrm{C}$ and wound infections.

The most frequent complications in the lymphadenectomy group were abdominal haemorrhage (3.35\%) and wound healing complications (3.35\%). The difference between the groups was statistically significant $(\mathrm{p}=0.002)$.

Due to postoperative complications in the lymphadenectomy group, reoperative procedures were necessary in $15(7.11 \%)$ patients. The difference was statistically significant $(\mathrm{p}=0.000)$.

The results of our analysis are partially in agreement with those of a study by Panici et al $(3,9)$. One of the aims of the study by Panici et al was the assessment of perioperative complications. The investigators confirmed that the median time of surgery was longer by approximately $90 \mathrm{~min}$ in patients undergoing lymphadenectomy, and the median volume of blood loss was higher by approximately $350 \mathrm{ml}$, when compared to the group with no lymphadenectomy. The differences were statistically significant $(p=0.006)$. The frequency of the intraoperative complications was similar, while postoperative complications were more frequent in the lymphadenectomy group. The percentage of postoperative complications was as high as $28 \%$ in the lymphadenectomy group, whereas in the group without lymphadenectomy this value was $18 \%$. The most frequent complications were lymphoedemas and lymphatic cysts. Based on the obtained results, the authors suggested that, apart from the higher complication risk, lymphadenectomy, as part of the routine surgical treatment, was the beneficial option.

The complications involving lymphadenectomy in ovarian cancer patients are the subjects of various clinical studies $(3,5)$. This event supports the argument against this procedure being performed as a routine part of the surgical treatment for ovarian cancer patients. Subsequently, certain investigators emphasize the longer duration of surgery, the greater blood loss, and the risk of additional complications, when compared with the lymph node biopsy procedure only.

Byrom and Quentin (4) emphasize the need for future studies to determine the effect of lymphadenectomy on survival in light of the risk of complications.

Based on the results of the present analysis, it should be noted that, apart from the higher risk of complications in the lymphadenectomy group, overall and disease-free survival was longer in the lymphadenectomy group for the early ovarian cancer patients (FIGO I, II). On the other hand, overall and disease-free survival in the advanced ovarian cancer patients (FIGO III, IV) was found to be similar. This result is in accordance with the findings of Verleye et al (6).

Ceccaroni et al (10) reported lymphatic cyst formation, the risk of vessel and nerve damage, the extensive duration of the surgery and enhanced blood loss risk.

De Poncheville et al (11) disputed the therapeutic role of lymphadenectomy, particularly in early ovarian cancer. These authors stressed the fact that survival of patients with early ovarian cancer following adjuvant chemotherapy is satisfactory. The addition of the aortic lymphadenectomy procedure with no strong evidence of its beneficial effect, exposes these patients to the risk of complications. The authors proposed that for ovarian cancer patients, staged according to the FIGO classification as IC and IA-IB, G>1, that is, patients who are candidates for adjuvant chemotherapy treatment, lymphadenectomy should not be performed. However, future studies aimed at assessing the prognostic value of lymphadenectomy are required.

In contrast, findings of other studies have shown that the efficacy of chemotherapy is decreased in cases of lymph node 
metastases. It has been confirmed that penetration of chemotherapeutic agents into the retroperitoneal space is limited and that cancer cells located in the lymph nodes are usually diploid and in the S-cell cycle phase. Therefore, these agents are less sensitive to cytostatic drug action $(5,12,13)$.

In order to reduce the risk of complications, Dobbs and Williamson (14) proposed laparoscopic lymphadenectomy for ovarian cancer patients. However, these authors emphasized that this procedure should be considered for early ovarian cancer patients and that confirmation of its therapeutic value requires future investigation.

In the investigation of the therapeutic value of lymphadenectomy in ovarian cancer patients, a large majority of investigators focused on other factors that should be considered during the analysis. These include factors related to the patient, such as age $(2,15)$ and coexistent diseases $(10)$, as well as factors related to the tumor, such as the clinical stage of the disease, and human factors, such as the proper treatment decision and quality of the surgical procedure performed (1,2,16-19).

In their study, Bidzinski et al (20) reported surgery-related complications in the two types of surgical treatment, in advanced ovarian cancer patients (FIGO III-IV). The difference in the survival of patients in the two groups did not achieve statistical significance (20). However, the observation that survival in the two groups was similar, following an analysis of the two types of surgical treatment in advanced ovarian cancer patients without lymphadenectomy, is significant.

These results are similar to those of the analysis presented in this study. Although the difference in the frequency and nature of postsurgical complications was statistically significant, the survival difference between the groups with and without lymphadenectomy in advanced ovarian cancer was not demonstrated.

Apart from the higher complication risk related to lymphadenectomy, the overall and disease-free survival in early ovarian cancer patients was prolonged when compared with the group without this procedure.

The application of aortic and pelvic lymphadenectomy, as a routine procedure for ovarian cancer patients, requires future randomized trials to demonstrate its prognostic value.

\section{References}

1. Trope $\mathrm{C}$ and Kaern J: Primary surgery for ovarian cancer. Eur J Surg Oncol 32: 844-852, 2006.

2. Aletti GD, Dowdy S, Podratz KC, et al: Role of lymphadenectomy in the management of grossly apparent advanced stage epithelial ovarian cancer. Am J Obstet Gynecol 195: 1862-1868, 2006.
3. Panici BP, Maggioni A, Hacker N, et al: Systematic aortic and pelvic lymphadenectomy versus resection of bulky nodes only in optimally debulked advanced ovarian cancer: a randomized clinical trial. J Natl Cancer Inst 97: 560-566, 2005.

4. Byrom J and Quentin D: Cancer of the ovary. Obstet Gynecol 13: 88-94, 2003

5. Angioli R, Plotti F, Palaia I, et al: Update on lymphadenectomy in early and advanced ovarian cancer. Obstet Gynecol 20: 34-39, 2008.

6. Verleye L, Ottevanger PB, van der Graaf W, et al: EORTG-GCG process quality indicators for ovarian cancer surgery. Eur J Cancer 45: 517-526, 2009.

7. Ayhan A, Gultekin M, Dursun P, et al: Metastatic lymph node number in epithelial ovarian carcinoma: does it have any clinical significance? Gynecol Oncol 108: 428-432, 2008.

8. Maggioni A, Panici PB, Dell'Anna T, et al: Randomized study of systematic lymphadenectomy in patients with epithelial ovarian cancer macroscopically confined to the pelvis. Br J Cancer 95: 699-704, 2006.

9. Panici PB, Maggioni A, Hacker N, et al: Systematic aortic and pelvic lymphadenectomy prolonged progression-free survival in advanced ovarian cancer. Gynecol Oncol 7: 209-210, 2005.

10. Ceccaroni M, D'Agostino G, Ferrandina G, et al: Gynecological malignancies in elderly patients: is age 70 a limit to standard-dose chemotherapy? An Italian Retrospective Toxicity Multicentric Study. Gynecol Oncol 85: 445-450, 2002.

11. De Poncheville L, Perrotin F, Lefrancq T, et al: Does paraaortic lymphadenectomy have a benefit in the treatment of ovarian cancer that is apparently confined to the ovaries? Eur J Cancer 37: 210-215, 2001.

12. Chambers SK: Systematic lymphadenectomy in advanced epithelial ovarian cancer: two decades of uncertainty resolved. J Natl Cancer Inst 97: 548-549, 2005.

13. Chan JK, Munro EG, Cheung MK, et al: Association of lymphadenectomy and survival in stage I ovarian cancer patients. Obstet Gynecol 109: 12-19, 2007.

14. Dobbs S and Wiliamson K: Laparoscopic lymphadenectomy in gynecological oncology. Obstet Gynecol 8: 109-112, 1998.

15. Du Bois A, Reuss A, Pujade-Lauraine E, et al: Role of surgical outcome as prognostic factor in advanced epithelial ovarian cancer: a combined exploratory analysis of 3 prospectively randomized phase 3 multicenter trials: by the Arbeitsgemeinschaft Gynaekologische Onkologie Studiengruppe Ovarialkarzinom (AGO-OVAR) and the Groupe d'Investigateurs Nationaux Pour les Etudes des Cancers de l'Ovarie (GINECO). Cancer 115: 1234-1244, 2009.

16. Chan JK, Urban R, Hu JM, et al: The potential therapeutic role of lymph node resection in epithelial ovarian cancer: a study of 13918 patients. Br J Cancer 96: 1817-1822, 2007.

17. Stratton JF, Tiddy JA and Paterson ME: The surgical management of ovarian cancer. Cancer Treat Rev 27: 111-118, 2001.

18. Ushijma K: Management of retroperitoneal lymph nodes in the treatment of ovarian cancer. Int J Clin Oncol 12: 181-186, 2007.

19. Oksefjell H, Sandstad B and Trope C: Ovarian cancer stage IIIC. Consequences of treatment level on overall and progression-free survival. Eur J Gynaecol Oncol 27: 209-214, 2006.

20. Bidzinski M, Derlatka P, Kubik P, et al: The evaluation of intra and postoperative complications related to debulking surgery with bowel resection in patients with FIGO stage III-IV ovarian cancer. Int J Gynecol Cancer 17: 993-997, 2007. 